\title{
Classification and regression tree model for predicting tracheostomy in patients with traumatic cervical spinal cord injury
}

\author{
Dae-Sang Lee ${ }^{1} \cdot$ Chi-Min Park ${ }^{2,3} \cdot$ Keumhee Chough Carriere ${ }^{4,5} \cdot$ Joonghyun Ahn $^{4}$
}

Received: 21 October 2015/Revised: 1 April 2017/Accepted: 17 April 2017/Published online: 26 April 2017

(C) Springer-Verlag Berlin Heidelberg 2017

\begin{abstract}
Purpose In patients with cervical spinal cord injury (CSCI), respiratory compromise and the need for tracheostomy are common. The purpose of this study was to identify common risk factors for tracheostomy following traumatic CSCI and develop a decision tree for tracheostomy in traumatic CSCI patients without pulmonary function test.

Methods Data of 105 trauma patients with CSCI admitted in our institution from April, 2008 to February, 2014 were retrospectively analyzed. Patients who underwent tracheostomy were compared to those who did not. Stepwise logistic regression analysis and classification and regression tree model were used to predict the risk factors for tracheostomy.

Results Tracheostomy was performed in $20 \%$ of patients with traumatic CSCI on median hospital day 4. Patients who underwent tracheostomy tended to be more severely injured (higher Injury Severity Score, lower Glasgow
\end{abstract}

Chi-Min Park

dr99.park@samsung.com

1 Department of Critical Care Medicine, Kangbuk Samsung Hospital, Sungkyunkwan University School of Medicine, Seoul, Republic of Korea

2 Department of Critical Care Medicine, Samsung Medical Center, Sungkyunkwan University School of Medicine, 81 Irwon-ro, Gangnam-gu, Seoul 135-710, Republic of Korea

3 Department of Surgery, Samsung Medical Center, Sungkyunkwan University, School of Medicine, Seoul, Republic of Korea

4 Biostatistics and Clinical Epidemiology Center, Samsung Medical Centre, Seoul, Republic of Korea

5 Department of Mathematical and Statistical Sciences, University of Alberta, Edmonton, AB, Canada
Coma Score, and lower systolic blood pressure on admission) which required more frequent intubation in the emergency room (ER) with a higher rate of complete CSCI compared to those who did not. Upon multiple logistic analysis, Age $\geq 55$ years (OR: 6.86, $p=0.037$ ), Car accident (OR: 5.8, $p=0.049$ ), injury above C5 (OR: $28.95, p=0.009$ ), ISS $\geq 16$ (OR: $12.6, p=0.004)$, intubation in the ER (OR: 23.87, $p=0.001$ ), and complete CSCI (OR: 62.14, $p<0.001$ ) were significant predictors for the need of tracheostomy after CSCI. These factors can predict whether a new patient needs future tracheostomy with $91.4 \%$ accuracy.

Conclusions Age $\geq 55$ years, injury above C5, ISS $\geq 16$, Car accident, intubation in the ER, and complete CSCI were independently associated with tracheostomy after CSCI. CART analysis may provide an intuitive decision tree for tracheostomy.

Keywords Tracheostomy $\cdot$ Trauma $\cdot$ Cervical spinal cord injury

$\begin{array}{ll}\text { Abbreviations } \\ \text { AIS } & \begin{array}{l}\text { American spinal injury association Impairment } \\ \text { Scale }\end{array} \\ \text { CART } & \text { Classification and regression tree } \\ \text { CSCI } & \text { Cervical spinal cord injury } \\ \text { ER } & \text { Emergency room } \\ \text { GCS } & \text { Glasgow Coma Scale } \\ \text { ICU } & \text { Intensive care unit } \\ \text { ISS } & \text { Injury severity score } \\ \text { IQR } & \text { Interquartile ranges } \\ \text { OR } & \text { Odds ratio } \\ \text { SBP } & \text { Systolic blood pressure } \\ \text { SCI } & \text { Spinal cord injury }\end{array}$




\section{Introduction}

Traumatic cervical spinal cord injury (CSCI) is a devastating and potentially life-threatening injury. The majority of early deaths following acute traumatic tetraplegia are due to pulmonary complications resulting from various inspiratory and/or expiratory muscle paralysis as well as excessive bronchial mucous production [14, 25]. Patients with traumatic CSCI are commonly intubated in the emergency department. Some of them require tracheostomy in spite of intensive care. Tracheostomy is more comfortable than an endotracheal tube. The beneficial effects of early tracheostomy in trauma patients have been reported $[1,3,6]$. Many studies have predicted the risk factors for tracheostomy in traumatic CSCI. Age, comorbidity, level of injury (neurological level), extent of injury (complete/incomplete), and forced vital capacity (FCV) are reported to be risk factors of CSCI. Recently, Yugue et al. [25] and Berney et al. [4] have analyzed clinical decisions to perform tracheostomy for patients with traumatic CSCI using a classification and regression tree (CART). They have reported that FVC is the first node in the tree. However, in the real world, it is difficult to measure FVC of patients with traumatic CSCI during acute phase. Therefore, the objective of this study was to identify common risk factors for tracheostomy following traumatic CSCI and develop a decision tree for tracheostomy in traumatic CSCI patients without pulmonary function test.

\section{Methods}

\section{Study population}

This was a retrospective single-center study conducted at a university affiliated-tertiary referral hospital in South Korea from April, 2008 to February, 2014. We retrospectively identified patients aged 18 years or older with traumatic CSCI who were admitted to the emergency department through an automated query of our hospital's database. CSCI was confirmed based on electronic medical records review. Exclusion criteria were: death within $24 \mathrm{~h}$, traumatic brain injury defined as intracranial hemorrhage on computed tomography, patients who were transferred to another hospital from our emergency room, and simple spinal sprain without spinal fracture or spinal cord injury. The local Institutional Review Board approved this study and waived the requirement for informed consent (IRB No. 2015-07-033-001) due to its retrospective nature.

\section{Data collection}

Data were extracted from medical records, including age, sex, Injury Severity Score (ISS), Glasgow Coma Scale (GCS), mechanism of injury, trauma associated injury, physiologic variables, laboratory variables, and intubation related variables. Pulmonary complication was defined as pneumonia, complicated pleural effusion, and atelectasis requiring additional oxygen supply. The degree of spinal cord injury was based on neurological examination using the American spinal injury association Impairment Scale (AIS) [15]. Respiratory impairment was defined as a respiratory rate of less than $10 / \mathrm{min}$ or more than $24 / \mathrm{min}$. Hypotension was defined as an initial systolic blood pressure of less than $90 \mathrm{mmHg}$ at the emergency department. Chest injury was defined as pneumothorax, hemothorax, or frail chests. Abdominal injury was defined as solid organ injury $[18,19]$ above grade 2 with visceral organ injury that required operation or pelvic bone fracture. Tracheostomy within 7 days after the accident was defined as early tracheostomy.

\section{Statistical analysis}

All variables are presented as median and interquartile ranges (IQRs) or as the number (percentage) of patients. For continuous variables, data were compared with MannWhitney $U$ test. For categorical variables, data were compared with the Chi-square test or Fisher's exact test. Stepwise logistic regression analysis was used to control the effects of confounding variables to identify independent risk factors for tracheostomy. To describe the decision mechanism, we performed classification and regression tree (CART) analysis using the recursive partitioning method [9, 10, 12]. All statistical analyses were performed using SPSS 20.0 (SPSS Inc., Chicago, IL, USA) and R 3.0.2, (R Foundation for Statistical Computing, Vienna, Austria).

\section{Results}

One hundred and five patients with traumatic CSCI were included in the analysis. Tracheostomy was performed in $21(20 \%)$ patients with traumatic CSCI. There were 86 (82\%) males. The median time from the accident to tracheostomy was 6 days (IQR, 2-12). Performance of tracheostomy based on AIS grade is delineated in Table 1 . Respiratory complication was more common with AIS grade A compared to AIS grade B-D. Demographics of patients with or without tracheostomy are summarized in Table 2. Patients with tracheostomy tended to have higher 
Table 1 Comparisons of clinical characteristics of patients according to AIS grade

\begin{tabular}{|c|c|c|c|c|}
\hline & AIS A $(n=17)$ & AIS B $(n=9)$ & AIS C $(n=31)$ & AIS D $(n=48)$ \\
\hline Age (years) & $30(28-56)$ & $64(55-72)$ & $57(49-66)$ & $51(31-61)$ \\
\hline Sex (male), $n(\%)$ & $14(82.4)$ & $6(66.7)$ & $31(100)$ & $35(72.9)$ \\
\hline ISS, median (IQR) & $16(16-20)$ & $9(9-16)$ & $9(7-14)$ & $9(9-12)$ \\
\hline GCS, median (IQR) & $14(10-15)$ & $15(14-15)$ & $15(14-15)$ & $15(14-15)$ \\
\hline Length of hospital stay (days), median (IQR) & $66(45-83)$ & $80(58-92)$ & $38(28-55)$ & $13(10-22)$ \\
\hline Length of ICU stay (days), median (IQR) & $12(3-18)$ & $15(3-19)$ & $0(0-4)$ & $0(0-0)$ \\
\hline Tracheostomy, $n(\%)$ & $13(76.5)$ & $4(44.4)$ & $4(12.9)$ & $0(0)$ \\
\hline Pre-tracheostomy pulmonary complication, $n(\%)$ & $6(35.3)$ & $1(11.1)$ & $0(0)$ & $0(0)$ \\
\hline Pulmonary complication during hospitalization, $n(\%)$ & $12(70.6)$ & $5(55.6)$ & $3(9.7)$ & $1(2.1)$ \\
\hline
\end{tabular}

GCS Glasgow Coma Scale, ICU intensive care unit, ISS injury severity score

Table 2 Clinical characteristics of patients in the tracheostomy and non-tracheostomy groups

\begin{tabular}{|c|c|c|c|}
\hline & Non-tracheostomy $(n=84)$ & Tracheostomy $(n=21)$ & $p$ value \\
\hline Age (years) & $54(42-62)$ & $47(29-70)$ & 0.62 \\
\hline Age $\geq 55$ (years) & $41(48.8)$ & $9(42.9)$ & 0.63 \\
\hline Sex (male) & $68(81.0)$ & $18(85.7)$ & 0.76 \\
\hline Car accident, $n(\%)$ & $38(45.2)$ & $11(66.7)$ & 0.079 \\
\hline Intubation in emergency room, $n(\%)$ & $3(3.6)$ & $11(52.4)$ & $<0.001$ \\
\hline Respiratory impairment, $n(\%)$ & $4(4.8)$ & $2(9.5)$ & 0.35 \\
\hline Hypotension, $n(\%)$ & $6(7.1)$ & $7(33.3)$ & 0.004 \\
\hline $\mathrm{GCS} \leq 8, n(\%)$ & $3(3.6)$ & $5(23.8)$ & 0.008 \\
\hline $\mathrm{ISS} \geq 16, n(\%)$ & $18(21.4)$ & $17(81.0)$ & $<0.001$ \\
\hline Chest injury, $n(\%)$ & $3(3.6)$ & $1(4.8)$ & $>0.99$ \\
\hline Abdominal/pelvic injury, $n(\%)$ & $2(2.4)$ & $1(4.8)$ & 0.49 \\
\hline Complete SCI (AIS A), $n(\%)$ & $4(4.8)$ & $13(61.9)$ & $<0.001$ \\
\hline Impaired motor function, $n(\%)$ & $9(10.7)$ & $17(81.0)$ & $<0.001$ \\
\hline Injury above $\mathrm{C} 5, n(\%)$ & $63(75.0)$ & $20(95.2)$ & 0.068 \\
\hline Spinal surgery, $n(\%)$ & $61(72.6)$ & $19(90.5)$ & 0.086 \\
\hline Duration of accident to tracheostomy creation (days), median (IQR) & NA & $6(2-7)$ & NA \\
\hline Length of hospital stay (days), median (IQR) & $23(11-42)$ & $75(45-91)$ & $<0.001$ \\
\hline Length of ICU stay (days), median (IQR) & $0(0-1)$ & $16(9-26)$ & $<0.001$ \\
\hline
\end{tabular}

AIS American spinal injury association Impairment Scale, GCS Glasgow Coma Scale, ICU intensive care unit, ISS injury severity score, $S B P$ systolic blood pressure, SCI spinal cord injury

ISS and lower Glasgow Coma Scale (GCS) with systolic blood pressure (SBP) $<90 \mathrm{mmHg}$ on admission compared to those without tracheostomy. The occurrence of impaired motor function was higher in patients who underwent tracheostomy than in patients without tracheostomy. The rate of intubation at the emergency room was also higher in patients who underwent tracheostomy. However, injury above $\mathrm{C} 5$ and respiratory impairment were not statistically different between patients with tracheostomy and those without tracheostomy. The mechanism of trauma (car accident versus non-car accident), chest injury, and abdominal injury was not significantly different between the two groups. A total of $80(76.2 \%)$ TCSCI patients underwent surgeries, including $19(23.8 \%)$ patients who underwent tracheostomy. The mean time from trauma to tracheostomy was 7.2 days. Eight patients underwent surgery and tracheostomy simultaneously with a mean time from trauma to surgery and tracheostomy of 2.9 days. However, seven patients underwent tracheostomy after the surgery with a mean time from trauma to surgery of 
1.4 days and a mean period from trauma to tracheostomy of 11.3 days.

The clinical characteristics of ten patients who underwent tracheostomy within 7 days (early tracheostomy group) and 11 patients who received tracheostomy after 7 days (late tracheostomy group) are shown in Table 3. More patients suffered from pneumonia before tracheostomy in the late tracheostomy group compared to those in the early tracheostomy group (60.0 vs $9.1 \%$, $p=0.024)$. However, overall pneumonia incidence during hospitalization was not statistically different between the two groups $(70.0$ vs $45.5 \%, p=0.39)$. Length of mechanical ventilator support, length of stay in the intensive care unit, and length of hospital stay were not significantly different between the early tracheostomy group and the late tracheostomy groups. Successful tracheostomy weaning at hospital discharge was not significantly different between the two groups either.

Multiple logistic regression results predicting tracheostomy are summarized in Table 4. Logistic regression identified age $\geq 55$ years [odds ratio (OR) $6.86,95 \% \mathrm{CI}$ $1.11-71.50 ; p=0.037$ ], injury above C5 (OR 28.95, 95\%
CI 2.01-9277.55; $p=0.009$ ), ISS $\geq 16$ (OR 12.64, 95\% CI $2.13-131.85 ; p=0.004)$, intubation at the emergency room (OR 23.87, 95\% CI 3.22-346.70; $p=0.001$ ), car accident (OR 5.80, 95\% CI 1.01-59.72, $p=0.049$ ), and AIS grade A (OR 62.14, 95\% CI 7.73-1541.92; $p<0.001$ ) as independent risk factors for tracheostomy in patients with traumatic CSCI. The high odds ratios in these risk factors indicated extreme skewness of data between the two groups under consideration. For example, among 17 patients in AIS grade A, $13(76 \%)$ had tracheostomy, while only $8(9.1 \%)$ out of 88 patients not in the AIS grade A category underwent tracheostomy procedure. The naïve OR was 32.5. After adjusting for other factors and removing small sample biases via Firth's method [8], the OR was estimated to be 62.14. AIS grade A was a highly significant indicator for tracheostomy. This was also evident in the CART analysis shown below. In our analysis, AIS grade A was the most significant criterion for dividing and determining patients who required tracheostomy, followed by whether ER intubation was performed or not. Specifically, if a patient had an AIS grade A, he or she was classified as requiring tracheostomy without a doubt, while

Table 3 Comparison of clinical characteristics according to early tracheostomy and late tracheostomy

\begin{tabular}{|c|c|c|c|}
\hline & Early tracheostomy $(n=11)$ & Late tracheostomy $(n=10)$ & $p$ value \\
\hline Age (years) & $47(29-72)$ & $49(30-56)$ & 0.72 \\
\hline Age $\geq 55$ & $5(45.5)$ & $4(40.0)$ & $>0.999$ \\
\hline Sex (male) & $10(90.9)$ & $8(80.0)$ & 0.59 \\
\hline Car accident, $n(\%)$ & $9(81.8)$ & $5(50.0)$ & 0.18 \\
\hline Intubation in emergency room, $n(\%)$ & $4(36.4)$ & $7(70.0)$ & 0.20 \\
\hline Respiratory impairment, $n(\%)$ & $0(0.0)$ & $2(20.0)$ & 0.21 \\
\hline Hypotension, $n(\%)$ & $5(45.5)$ & $2(20.0)$ & 0.36 \\
\hline $\mathrm{GCS} \leq 8, n(\%)$ & $8(72.7)$ & $8(80.0)$ & $>0.99$ \\
\hline ISS $\geq 16, n(\%)$ & $9(81.8)$ & $8(80.0)$ & $>0.99$ \\
\hline Chest injury, $n(\%)$ & $1(9.1)$ & $0(0.0)$ & $>0.99$ \\
\hline Abdominal/pelvic injury, $n(\%)$ & $0(0.0)$ & $1(10.0)$ & 0.48 \\
\hline Complete SCI (AIS A), $n(\%)$ & $5(45.5)$ & $8(80.0)$ & 0.18 \\
\hline Impaired motor function, $n(\%)$ & $8(72.7)$ & $9(90.0)$ & 0.59 \\
\hline Injury above $\mathrm{C} 5, n(\%)$ & $11(100.0)$ & $9(90.0)$ & 0.48 \\
\hline Pre-tracheostomy respiratory complications, $n(\%)$ & $1(9.1)$ & $6(60.0)$ & 0.024 \\
\hline Respiratory complications during hospitalization, $n(\%)$ & $5(45.5)$ & $7(70.0)$ & 0.39 \\
\hline Spinal surgery, $n(\%)$ & $10(90.9)$ & $9(90.0)$ & $>0.99$ \\
\hline Duration of accident to tracheostomy creation (days), median (IQR) & $3(1-5)$ & $12(8-14)$ & $<0.001$ \\
\hline MV day (days), median (IQR) & $9(2-13)$ & $10(8-17)$ & 0.27 \\
\hline Length of hospital stay (days), median (IQR) & $75(39-80)$ & $78(63-117)$ & 0.25 \\
\hline Length of ICU stay (days), median (IQR) & $15(7-18)$ & $18(10-41)$ & 0.26 \\
\hline Successful tracheostomy weaning at hospital discharge, $n(\%)$ & $7(63.6)$ & $6(60)$ & $>0.99$ \\
\hline
\end{tabular}

AIS American spinal injury association Impairment Scale, GCS Glasgow Coma Scale, ICU intensive care unit, ISS injury severity score, SBP systolic blood pressure, $S C I$ spinal cord injury 
Table 4 Predictors for tracheostomy in patients with spinal cord injury after stepwise logistic regression

\begin{tabular}{lccrr}
\hline & \multicolumn{3}{l}{$95 \% \mathrm{CI}$} & \\
\cline { 2 - 5 } & Adjusted OR & Lower & Upper & $p$ value \\
\hline Age $\geq 55$ (years) & 6.86 & 1.12 & 71.50 & 0.037 \\
Injury above C5 & 28.95 & 2.01 & 9277.55 & 0.009 \\
Car accident & 5.80 & 1.01 & 59.72 & 0.049 \\
ISS $\geq 16$ & 12.64 & 2.13 & 131.85 & 0.004 \\
Intubated at emergency room & 23.87 & 3.22 & 346.70 & 0.001 \\
Complete CSCI (AIS A) & 62.14 & 7.73 & 1541.92 & $<0.001$ \\
\hline
\end{tabular}

Small sample biases were adjusted by the Firth's penalized method. The high odds ratios in these risk factors indicate extreme skewness of the data between the two groups under consideration a patient with AIS grades other than A was classified as requiring tracheostomy if he or she had an intubation in the ER. The cross-validated misclassification rate obtained from Lachenbruch's hold out procedure was $12.2 \%$. Therefore, the estimated correct classification rate was $87.8 \%$. Detailed path analysis for the classification and estimation of probability of tracheostomy for each terminal node is shown in Fig. 1. On the other hand, if we used all significant variables selected from stepwise logistic regression, the cross-validated error rate was improved to $8.6 \%$ for non-hierarchical decision making for tracheostomy. The improvement was an incorrect prediction for only three patients who underwent tracheostomy compared to CART's incorrect prediction of seven patients. According to the logistic regression, the chances of undergoing tracheostomy were increased for patients who were older than 55 , with an injury above $\mathrm{C} 5$, had a car accident, or had an ISS score over 16, in addition to their AIS grade status and whether they received intubation at the ER.

\section{Discussion}

In our study, we found that six factors $(\mathrm{GCS} \leq 8$, ISS $\geq 16$, SBP $<90 \mathrm{mmHg}$, intubation at the emergency department, AIS grade A, and injury above C5) were independent risk factors for tracheostomy following traumatic CSCI in univariate analysis. In addition, ISS $\geq 16$, intubation at the emergency department, AIS grade A, and injury above $\mathrm{C} 5$ were found to be independent risk factors

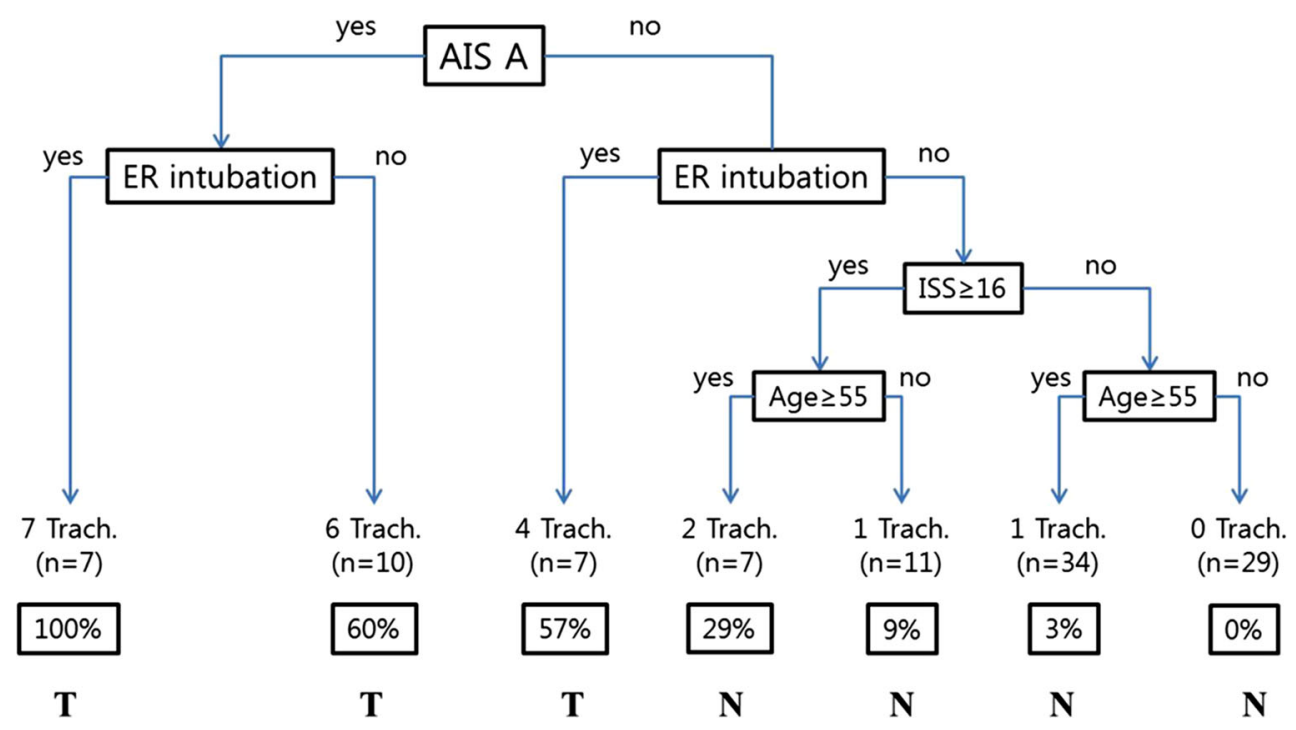

Fig. 1 Decision tree model for tracheostomy in patients with traumatic cervical spinal cord injury. The boxes denote discriminating variables from CART analysis. Figure depicts how the decision for tracheostomy is made. At the bottom of each branch, the actual \% of tracheostomy performed is indicated. The bottom line shows the decision tree results ( $T$ tracheostomy, $N$ no tracheostomy). The CART classifies all patients of AIS grade A as requiring tracheostomy. When

AIS grades are other than grade A, patients are classified as requiring tracheostomy if intubation is performed at the ER. Otherwise, no tracheostomy is required. This simple classification has a crossvalidated corrected classification rate of $87.8 \%$. AIS American spinal injury association Impairment Scale, ER emergency room, trach tracheostomized patient 
for tracheostomy following traumatic CSCI in multivariate analysis.

Patients classified as AIS grade A were found to have a higher likelihood of tracheostomy compared to others with AIS grade B-D in this study. This finding supports the results of other studies suggesting that complete SCI is a risk factor for the need of tracheostomy $[11,16,17,20,25]$. Nevertheless, we should keep variability in mind, because $35 \%$ of AIS grade A patients did not receive tracheostomy during hospitalization. Therefore, the decision to undergo tracheostomy should not be simply based on the severity of spinal cord injury graded by the AIS score. According to our CART analysis, it may be useful to consider whether the patients have undergone an intubation in the emergency department.

The level of CSCI is a very simple and useful indicator for predicting the need for tracheostomy in patients with traumatic CSCI [5, 7, 13, 16]. Since the phrenic nerve arises from C3 through C5 spinal nerve, diaphragmatic dysfunction is inevitable in patients with injury above C5. CSCI below C5 does not involve the phrenic nerve. Thus, a patient with such an injury can still breathe in spite of possible paralysis of the lower limb. In this study, patients with injury above C5 were found to have a higher likelihood of tracheostomy compared to those with injury below C6. Branco et al. [5] have recommend that patients with injury above C5 should be considered for tracheostomy, while McCully et al. [16] have recommend that patients with injury above C6 should be considered for tracheostomy. Clinically, it is hard to determine the precise level of CSCI at acute stage. Consequently, the decision to perform tracheostomy by only depending on the level of CSCI is difficult. In our CART analysis, the level of CSCI was pushed back on the priority list.

ISS is an anatomical base scoring system that describes patient severity by the overall score of injured sites [2]. Patients with higher ISS required tracheostomy and intensive treatment for respiratory complications in several studies [5, 17, 25]. ISS was a good predictor for tracheostomy as well as a good indicator for the severity of the injury in these previous studies. However, some studies do not recommend the use of ISS for predicting the need for tracheostomy [16, 22]. Precise ISS scoring within 2 days after injury is not feasible.

McCully et al. [16] have reported that there is no significant relationship between age and the need for tracheostomy. However, some studies have reported a significant relationship between the two. Yugue et al. [25] have found that being older than 69 years is a risk factor for tracheostomy in multivariate analysis. Wicks et al. [23] have found that being older than 50 years is a risk factor for ventilator dependency. Our study showed a significant relationship between age and the need for tracheostomy in multivariate analysis.
Some studies have reported the benefits of early tracheostomy in the CSCI patients [1, 6]. However, in our study, there was no clear benefit of performing the early tracheostomy compared to performing late tracheostomy. Early tracheostomy did not prevent respiratory complications during hospitalization. The length of mechanical ventilator support, ICU length of stay, and hospital length of stay were not statistically different between the two groups (early tracheostomy and late tracheostomy).

According to Wilson et al., the patients with facet injury are likely to have an SCI [24]. Recently, the new classification system based on morphology type with additional descriptions for facet injuries was proposed by the AOSpine Knowledge forum [21]. Although we did not use the AOSpine classification in this study, if AOSpine classification was considered the later study, it will help predict SCI and it would give more information on determining tracheostomy.

This study has several limitations. First, this was a retrospective and single-center study. Second, there was no standardized indication of intubation or tracheostomy in patients with traumatic CSCI. It was dependent on the primary physician or attending doctor in the emergency department. However, we adjusted for confounding factors such as age, trauma mechanism, respiratory impairment, and hypotension which were clues for deciding tracheostomy in a clinical setting. The strength of our study was that physicians were able to decide easily on whether to perform tracheostomy for patients with traumatic CSCI in the acute stage by using a decision tree. The weakness of our study which was common for classification tools was that it relied on derived rules, assuming that all influential variables were measured accurately. However, the environment and people are changing all the time. In addition, some key variables were not measurable. Therefore, although the estimated predictions were cross-validated, the misclassification error rate might be optimistic. Nevertheless, for patients with traumatic CSCI requiring the early tracheostomy, a prospective randomized trial might be able to identify optimal clinical guidelines. Several studies have shown the feasibility of decision making using CART for tracheostomy in TCSCI patients [4, 10, 25]. However, our study showed the simplest way to decide on whether tracheostomy should be performed without FVC or magnetic resonance imaging.

In conclusion, the following six risk factors were found to be able to predict tracheostomy after traumatic CSCI: age older than 55 years, ISS $\geq 16$, intubation at the emergency department, AIS grade A, car accident, and injury above C5. Although our data do not suggest that early tracheostomy can prevent respiratory complication, CART analysis can be used to provide a very intuitive decision tree for tracheostomy. 
Acknowledgements The authors thank Ms. Sookyoung Woo at Samsung Biomedical Research Institute for advice regarding statistical analysis. The authors also thank the Major Trauma Team for their enthusiasm and commitment to patient care.

Author contributions LDS and CMP contributed to the study design. LDS and CMP participated in data collection. DSL, JA, KCC, and CMP participated in the data analysis. All authors contributed to data interpretation. DSL, KCC, and CMP contributed to writing of the manuscript.

\section{Compliance with ethical standards}

\section{Funding/support None.}

\section{Financial disclosure None.}

Conflict of interest The authors declare that they have no competing interests.

\section{References}

1. Armstrong PA, McCarthy MC, Peoples JB (1998) Reduced use of resources by early tracheostomy in ventilator-dependent patients with blunt trauma. Surgery 124:763-766 (discussion 766-767)

2. Baker SP, O'Neill B, Haddon W Jr, Long WB (1974) The injury severity score: a method for describing patients with multiple injuries and evaluating emergency care. J Trauma 14:187-196

3. Ball PA (2001) Critical care of spinal cord injury. Spine (Phila Pa 1976) 26:S27-S30

4. Berney SC, Gordon IR, Opdam HI, Denehy L (2011) A classification and regression tree to assist clinical decision making in airway management for patients with cervical spinal cord injury. Spinal Cord 49:244-250

5. Branco BC, Plurad D, Green DJ, Inaba K, Lam L, Cestero R, Bukur M, Demetriades D (2011) Incidence and clinical predictors for tracheostomy after cervical spinal cord injury: a National Trauma Databank review. J Trauma 70:111-115

6. Brook AD, Sherman G, Malen J, Kollef MH (2000) Early versus late tracheostomy in patients who require prolonged mechanical ventilation. Am J Crit Care 9:352-359

7. Como JJ, Sutton ER, McCunn M, Dutton RP, Johnson SB, Aarabi B, Scalea TM (2005) Characterizing the need for mechanical ventilation following cervical spinal cord injury with neurologic deficit. J Trauma 59:912-916 (discussion 916)

8. Firth D (1993) Bias reduction of maximum likelihood estimates. Biometrika 80:27-38

9. Hastie T, Tibshirani R, Friedman J, Franklin J (2005) The elements of statistical learning: data mining, inference and prediction. Math Intell 27:83-85

10. Hou YF, Lv Y, Zhou F, Tian Y, Ji HQ, Zhang ZS, Guo Y (2015) Development and validation of a risk prediction model for tracheostomy in acute traumatic cervical spinal cord injury patients. Eur Spine J 24:975-984

11. Kornblith LZ, Kutcher ME, Callcut RA, Redick BJ, Hu CK, Cogbill TH, Baker CC, Shapiro ML, Burlew CC, Kaups KL,
DeMoya MA, Haan JM, Koontz CH, Zolin SJ, Gordy SD, Shatz DV, Paul DB, Cohen MJ (2013) Mechanical ventilation weaning and extubation after spinal cord injury: a Western Trauma Association multicenter study. J Trauma Acute Care Surg 75:1060-1069 (discussion 1069-1070)

12. Lachenbruch PA, Mickey MR (1968) Estimation of error rates in discriminant analysis. Technometrics 10:1-11

13. Leelapattana P, Fleming JC, Gurr KR, Bailey SI, Parry N, Bailey CS (2012) Predicting the need for tracheostomy in patients with cervical spinal cord injury. J Trauma Acute Care Surg 73:880-884

14. Lemons VR, Wagner FC Jr (1994) Respiratory complications after cervical spinal cord injury. Spine (Phila $\mathrm{Pa}$ 1976) 19:2315-2320

15. Marino RJ, Barros T, Biering-Sorensen F, Burns SP, Donovan WH, Graves DE, Haak M, Hudson LM, Priebe MM (2003) International standards for neurological classification of spinal cord injury. J Spinal Cord Med 26(Suppl 1):S50-S56

16. McCully BH, Fabricant L, Geraci T, Greenbaum A, Schreiber MA, Gordy SD (2014) Complete cervical spinal cord injury above C6 predicts the need for tracheostomy. Am J Surg 207:664-668 (discussion 668-669)

17. Menaker J, Kufera JA, Glaser J, Stein DM, Scalea TM (2013) Admission ASIA motor score predicting the need for tracheostomy after cervical spinal cord injury. J Trauma Acute Care Surg 75:629-634

18. Moore EE, Cogbill TH, Jurkovich GJ, Shackford SR, Malangoni MA, Champion HR (1995) Organ injury scaling: spleen and liver (1994 revision). J Trauma 38:323-324

19. Moore EE, Cogbill TH, Malangoni MA, Jurkovich GJ, Champion HR, Gennarelli TA, McAninch JW, Pachter HL, Shackford SR, Trafton PG (1990) Organ injury scaling, II: pancreas, duodenum, small bowel, colon, and rectum. J Trauma 30:1427-1429

20. Tanaka J, Yugue I, Shiba K, Maeyama A, Naito M (2016) A study of risk factors for tracheostomy in patients with a cervical spinal cord injury. Spine (Phila Pa 1976) 41:764-771

21. Vaccaro AR, Koerner JD, Radcliff KE, Oner FC, Reinhold M, Schnake KJ, Kandziora F, Fehlings MG, Dvorak MF, Aarabi B, Rajasekaran S, Schroeder GD, Kepler CK, Vialle LR (2016) AOSpine subaxial cervical spine injury classification system. Eur Spine J 25:2173-2184

22. Velmahos GC, Toutouzas K, Chan L, Tillou A, Rhee P, Murray J, Demetriades D (2003) Intubation after cervical spinal cord injury: to be done selectively or routinely? Am Surg 69:891-894

23. Wicks AB, Menter RR (1986) Long-term outlook in quadriplegic patients with initial ventilator dependency. Chest 90:406-410

24. Wilson JR, Vaccaro A, Harrop JS, Aarabi B, Shaffrey C, Dvorak M, Fisher C, Arnold P, Massicotte EM, Lewis S, Rampersaud R, Okonkwo DO, Fehlings MG (2013) The impact of facet dislocation on clinical outcomes after cervical spinal cord injury: results of a multicenter North American prospective cohort study. Spine (Phila Pa 1976) 38:97-103

25. Yugue I, Okada S, Ueta T, Maeda T, Mori E, Kawano O, Takao T, Sakai H, Masuda M, Hayashi T, Morishita Y, Shiba K (2012) Analysis of the risk factors for tracheostomy in traumatic cervical spinal cord injury. Spine (Phila Pa 1976) 37:E1633-E1638 